\title{
César Aguiar y la investigación social aplicada
}

\author{
Luis E. González (Uruguay)
}

César Aguiar, sociólogo y encuestador uruguayo, nació el 12 de julio de 1943 y murió el 30 de diciembre de 2011, a los sesenta y ocho años. Tenía sus achaques, que al final se impusieron, pero los ignoraba olímpicamente. Estaba hiperactivo, como siempre, y en la plenitud de sus recursos intelectuales. Más allá de los afectos de su familia y sus amigos, sus colegas en la profesión extrañaremos la ausencia de sus ideas y de su empuje. Aquí trato de explicar de manera concisa por qué razón lo extrañaremos.

Naturalmente, resumo mi visión personal de su historia. Visión que puede estar contaminada por las circunstancias, porque fui amigo suyo, aunque no sin altibajos. Nos conocimos antes del golpe de Estado de 1973 en el viejo Instituto de Ciencias Sociales de la Universidad de la República, perdimos contacto durante varios años, y desde entonces fuimos colegas, amigos, socios, nos divorciamos, estuvimos enemistados, luego fuimos competidores, y finalmente socios y amigos otra vez. Abreviando: de los últimos cuarenta años, casi las dos terceras partes estuvimos o bien sin contacto, o distanciados, o compitiendo de un modo activo. Todo esto tal vez me brinde una perspectiva equilibrada, aunque no la asegura. Sin embargo, creo que César Aguiar probablemente estaría de acuerdo con el grueso de lo que resumo a continuación.

Director de CIFRA, Director de SUMA. 
Aguiar fundó Equipos Consultores en Montevideo en 1976 (varios años después, Equipos MORI, también en Asunción y en Buenos Aires). En 1984 -fecha significativa y en absoluto accidental: la de la elección "de salida" del gobierno militar-, comenzó a trabajar en opinión pública en Montevideo. En el año 2010, fue uno de los cofundadores de Suma, una sociedad de tres partes (Equipos, Cifra y un pequeño grupo de amigos como socios inversores) dedicada a trabajar en la región. En Uruguay, Equipos y Cifra continuaron y continúan compitiendo.

Fue un intelectual católico, un intelectual a secas, y a mi modo de ver, uno de los principales impulsores del desarrollo de las ciencias sociales aplicadas en el Uruguay. Fue sociólogo, profesor, consultor, encuestador. No fundó las encuestas profesionales en el país: hubo otros precursores. El más conocido de ellos fue tal vez Luis Alberto Ferreira, que desde los años 1950 tuvo durante cerca de cuarenta años la representación de Gallup en el Uruguay.

Un rasgo distintivo de estos precursores (en Uruguay, aunque también en otros países de la región) fue su distancia respecto a su academia natural, la de las ciencias sociales. No era un pecado de distancia o abstención sólo de los precursores. Desde el mundo universitario (y más en general, desde la intelligentzia de la época), lo que hacían los precursores, si lo hacían honestamente, era para muchos una actividad sin vuelo alguno, de un materialismo banal, al servicio de Mammón (del mercado y de la lógica del lucro). Para peor, era dudoso que lo hicieran honestamente. Esta idea de la deshonestidad esencial de los encuestadores sobrevive hasta hoy, aunque amortiguada. ${ }^{2}$ Para el

1 En esos años, eran ideas bastante extendidas en la comunidad de las ciencias físico-naturales. En 1964, un distinguido físico (Premio Nobel en 1965) dijo: "En Estados Unidos, los dos partidos políticos han resuelto usar expertos en relaciones públicas, esto es, hombres anuncio que están entrenados en los métodos de decir la verdad y mentir para desarrollar 
ala más radical de la academia, estaban al servicio de un empirismo chato enemigo de la razón y del socialismo que se avizoraba en el futuro no muy lejano. Esto puede parecer hoy un tanto exagerado, pero estaba en el espíritu de los tiempos. En las condiciones y prácticas de la época, además, no siempre era fácil separar el trigo de la paja. Un ejemplo ayudará a ilustrar el punto.

Aguiar hizo algunas cosas con Ferreira. Los detalles pueden ser imprecisos (Aguiar me contó esta historia hace unos veinticinco años), pero lo esencial, en mi recuerdo, es lo que sigue. En los años '70 Ferreira debía hacer un relevamiento de la superficie industrial paraguaya, y lo hizo con Aguiar, no recuerdo en qué circunstancias. Aguiar preparó una metodología prolija y detallada para obtener lo que querían, seguramente muy satisfactoria en principio, pero inviable con los recursos que tenían. Ferreira le dijo entonces: "Mire, Aguiar, los predios industriales tienen que estar sobre una ruta transitable que soporte camiones. En Paraguay hay básicamente dos rutas importantes asfaltadas. Alquilamos un auto y las recorremos". ¿Y entonces? ¿Al llegar a cada predio pedían una cita con el encargado, y le pedían la información del caso? Eso tampoco era posible: se necesitaría demasiado tiempo para resolver las previsibles e inevitables dificultades. Alquilaron el auto y comenzaron su recorrida, con Aguiar de copiloto, provisto de la papelería necesaria. Al llegar a una planta industrial, usualmente de base rectangular, es-

un producto". Diecisiete años después, en 1981, fue más general: "Las ciencias sociales son un ejemplo de una ciencia que no es ciencia [...]. No han llegado a ninguna parte todavía; quizá lleguen algún día [...]. Tenemos expertos en todo que parece que fueran expertos científicos. No son científicos... Hay todo tipo de mitos y pseudociencia por todas partes". (Richard Feynman, en 1964 en una conferencia en el Galileo Symposium en Italia, y en 1981, en una entrevista para la BBC, ambas reproducidas en una compilación de sus ensayos y entrevistas publicada como El placer de descubrir (Barcelona, Crítica, 2000, pp. 91 y 29, respectivamente). En la comunidad de las ciencias (o de las no ciencias) sociales, en cambio, las opiniones diferían mucho según los ámbitos y sus respectivas historias. En América Latina, podían ser muy duras. 
timaban a ojo las dimensiones (largo, ancho), y contaban el número de pisos, si los había. Con eso tenían una estimación de la superficie total, tal vez sin siquiera detener el auto, y al terminar la recorrida ya tenían el número que buscaban (y lo esencial del informe que debían). Aguiar, mucho más joven e inexperto que Ferreira, tenía sus dudas (y también sus temores). Pocos años después hubo un censo que midió lo que ellos habían buscado medir. La distancia entre el resultado del censo y la estimación de Aguiar y Ferreira resultó menos de un $10 \%$.

Esa historia condensa, a mi juicio, buena parte de las circunstancias de las "ciencias" sociales aplicadas. En ellas, no hay preguntas a secas, sino preguntas y precios (a fin de cuentas, como en las ingenierías). En un extremo de este espacio de preguntas y precios, algunas preguntas son relativamente fáciles y baratas de responder con cierta confianza. Por ejemplo: "En las elecciones presidenciales que se realizarán dentro de quince días, ¿hay algún ganador probable? ¿Quién, o quiénes? ¿Por qué?”. Hay luego una gradación compleja de preguntas, ordenables según la posible confianza en sus respuestas (cada vez menos), y también según sus precios (cada vez más, hasta llegar a los inaccesibles). Suele existir cierto trade off entre la confianza que merecen las respuestas y su precio. En el extremo opuesto a lo relativamente barato pero confiable, hay preguntas que aunque contaran con muchos recursos para buscar sus correspondientes respuestas, normalmente no sería posible contestarlas con razonable confianza. Por ejemplo, "en las elecciones presidenciales que se realizarán dentro de dos años, ¿hay algún ganador probable? ¿Quién, o quiénes? ¿Por qué?". Y sin embargo: aun así, los partidos necesitan candidatos, y necesitan apostar lo mejor que puedan, eligiendo las figuras con mejores posibilidades, porque sus opciones ayudarán o perjudicarán al propio partido. Esas figuras, los preprecandidatos, necesitan tomar decisiones 
difíciles (porque tienen consecuencias potencialmente pesadas sobre sus futuros personales). Si no es posible contestar a todos ellos con respuestas claras y confiables, al menos sería necesario brindarles conjeturas plausibles y fundamentadas. Esta es una de las tareas importantes de la investigación político-electoral aplicada. Pero no es fácil proveer con honestidad esta clase de respuestas. Son, inevitablemente, muy artesanales.

Parte del problema se puede apreciar examinando los límites de la comparación con las ingenierías. Una amplísima gama de las preguntas dirigidas a los ingenieros de hoy pueden ser contestadas (aunque a un cierto precio) porque se dispone de teorías robustas y muy generales que han soportado las pruebas de la crítica analítica y de la experiencia. Teorías que, para decirlo brevemente, no existen en las "ciencias" sociales (en eso creo que Feynman sigue teniendo razón). Los practicantes de estas últimas disciplinas se parecen más a los buenos herreros o armeros medievales que a los ingenieros contemporáneos. Sus productos son útiles y necesarios; cumplen las funciones que se espera de ellos. En su producción, acumulan tradiciones y experiencia, y usan algunas técnicas más o menos sistematizadas (las herramientas estadísticas, por ejemplo). Pero los armeros medievales no tenían -y los investigadores sociales contemporáneos, aplicados o no, tampoco tienenla clase de teorías generales ("universales") que sustentan el trabajo de los ingenieros.

Como entre los herreros medievales, en las ciencias sociales aplicadas también hay ahora acumulación y cierto progreso. Estas consideraciones permiten resumir cuál fue, a mi juicio, el aporte de César Aguiar a la profesión (o artesanía). Aguiar fue el primer sociólogo uruguayo que desde la academia tomó la posta de los primeros encuestadores, llevando las cosas un paso más adelante. Desde la creación de Equipos en 1976, su esfuerzo buscó 
deliberadamente insertar sus actividades en dos ámbitos más amplios. Primero, en la propia comunidad académica nacional. Esto impuso casi de manera espontánea rigor y transparencia. Luego, años después, en la comunidad profesional latinoamericana, esfuerzo que fructificó cuando por fin se fundó WAPOR Buenos Aires. ${ }^{3}$ La apertura que estos dos movimientos involucraban impuso estándares y prácticas que resultaban naturales para los que vinieron después, aun si sólo se preocupaban por su propio futuro. De esos dos grandes movimientos resultó una "desprovincianización" sistemática de la profesión. El esfuerzo fue pesado y largo: le dedicó treinta y cinco años de su vida. Pero creo que sus resultados son claros. Dos de ellos me parecen particularmente importantes desde esta perspectiva.

En primer lugar, cuando Aguiar empezó, no era en absoluto evidente que el ejemplo de más arriba (pronosticar el triunfador de una elección presidencial uruguaya con quince días de anticipación) fuera posible (menos aun fácil o barato). Cuarenta años después, todos o casi todos los interesados dan por sentado que eso es razonablemente fácil y barato (salvo en una elección muy pareja, pero esto sólo agrega una vuelta de tuerca adicional: es relativamente fácil y barato anticipar una elección pareja).

En segundo lugar, en Uruguay (y en muchos lugares de la región) las encuestas, en especial las políticas, nacieron con el estigma de la corrupción. Los encuestadores, se asumía, falseaban o directamente inventaban sus datos. Porque se vendían al mejor postor, o porque eran militantes disfrazados, pero falseaban o inventaban sus resultados. Los falseaban suponiendo que realmente los tuvieran, para lo cual era necesario gastar tiempo, esfuerzo y dinero en reunirlos más o menos de acuerdo con el canon. Si los tenían, usaban los datos verdaderos para sus propios fines, pero

World Association of Public Opinion Research en Buenos Aires. 
difundían (por un precio) resultados falsos: "salir primero", se solía suponer, cuesta tanto. Es posible que algunos aún lo crean. También podían abreviar ese esfuerzo y simplemente inventar los resultados: en Uruguay eso se llamaba (se sigue llamando), con delicadeza, "dibujar".

Sin duda hubo (y tal vez sigue habiendo) personajes que se dedicaron a estas actividades. En ocasiones, tripulan organizaciones "golondrinas" que aparecen y desparecen con las elecciones. Lo que importa, sin embargo, es su peso relativo dentro de la profesión; a fin de cuentas, todas las profesiones tienen sus manzanas podridas. En Uruguay, la imagen de una profesión monopolizada o dominada por las manzanas podridas fue cediendo lentamente a lo largo de los últimos veinticinco años. Me consta: esa ha sido mi experiencia personal. Pero el proceso tuvo consecuencias muy visibles. Junto con María Braun, presidenta de WAPOR Buenos Aires, en abril y mayo de 2010 realizamos una consulta vía Internet a miembros de la profesión y a otros académicos y especialistas usuarios de encuestas de opinión pública. ${ }^{4}$ Una de las preguntas inquiría por la seriedad que (a juicio de los consultados) sus respectivos públicos informados atribuían "a las encuestadoras más conocidas". Se recibieron respuestas de toda la región, y en seis países hubo un mínimo de respuestas (al menos una decena en cada uno) que permitían comparaciones preliminares: Argentina, Brasil, Chile, México, Perú y Uruguay. En conjunto, la mayoría absoluta de los consultados (55\%) opinó que la mayoría (o la gran mayoría) del público informado creía que esas encuestadoras eran serias, pero las respuestas diferían considerablemente entre los distintos países.

Los principales resultados de este estudio se encuentran en María Braun, "Encuestas de opinión pública en América Latina: desafíos y controversias", Revista Latinoamericana de Opinión Pública, Año 2011, Número 1, pp. 13-38. 
Uruguay estaba en un extremo: el $80 \%$ de los consultados sostuvo que la mayoría creía que las principales encuestadoras eran serias, y sólo el 7\% dijo que la mayoría pensaba que no eran serias (el resto veía "opiniones divididas"). Las opiniones de los consultados brasileños y mexicanos eran favorables a las encuestadoras y muy cercanas a las de los uruguayos. Los consultados chilenos, peruanos y argentinos, en cambio, veían mayoritariamente opiniones divididas. Pero mientras entre chilenos y peruanos muy pocos creían que la opinión informada consideraba que las encuestadoras no eran serias, en Argentina, el 37\% de los consultados pensaba de esa manera (y sólo el 17\% creía que las encuestadoras eran vistas como serias).

No pretendo decir que César Aguiar construyó él solo esos dos grandes resultados para la profesión en Uruguay. Sí creo que sin él, las cosas pudieron ser muy diferentes, y probablemente lo habrían sido. Si estas ideas son aproximadamente correctas, el verdadero papel de Aguiar en esta historia es el del innovador institucional, la figura que contribuye de manera decisiva a la emergencia y consolidación de algo nuevo. Y también sería claro que dejó huella: una huella importante por su contenido, por su forma de incrustarse en el desarrollo de las ciencias sociales (y de las ciencias sociales aplicadas) en el país y en la región, y por su relación con los debates ideológicos e intelectuales sobre la investigación social aplicada y las encuestas en particular que abundaron en las últimas décadas. Debates que todavía hoy nos siguen acompañando. Por todas estas razones, pienso que sus colegas en la investigación social, aplicada o no, extrañarán sus ideas y su empuje. 